
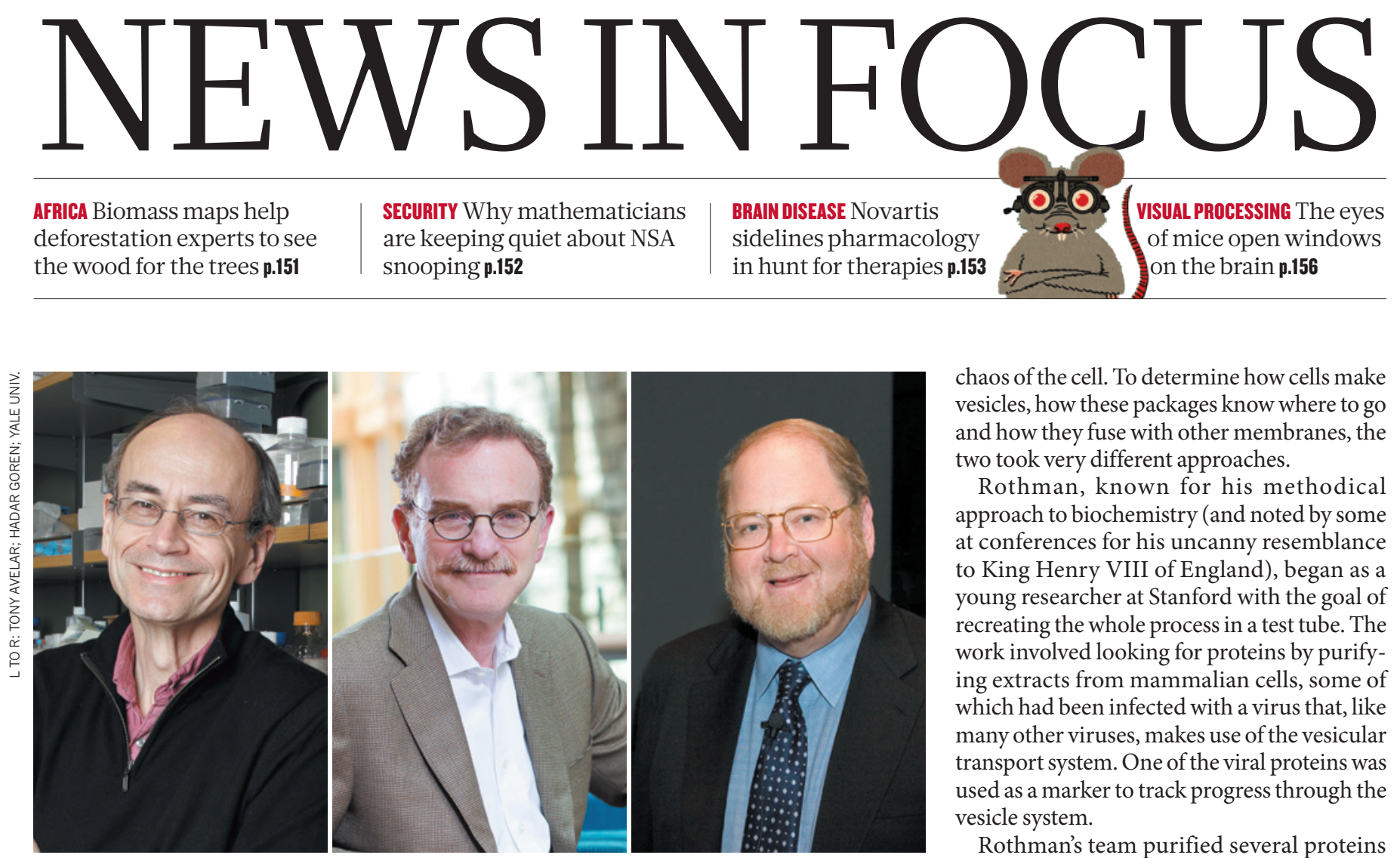

Thomas Südhof (left), Randy Schekman and James Rothman revealed how vesicles interact with targets.

\title{
NOBEL PRIZE
}

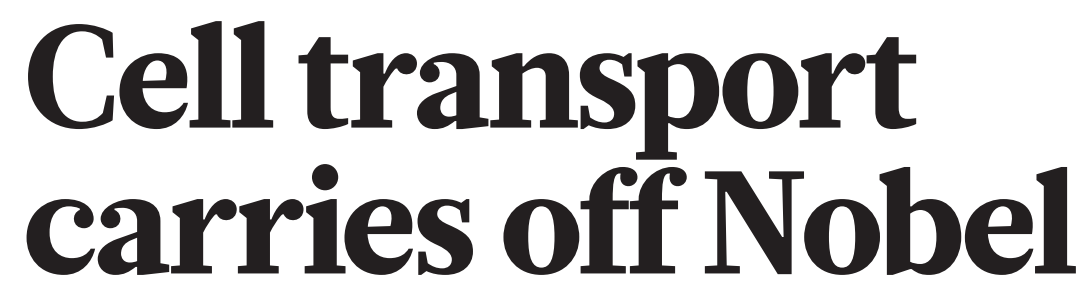

\section{Medicine prize goes to discoverers of vesicle system that shuttles biomolecules around cells.}

\section{BY EWEN CALLAWAY}

1 Three scientists who explained the inner workings of a 'cellular postal service' shared this year's Nobel Prize in Physiology or Medicine. Their work pinpointed how cells shuttle proteins and other biomolecules from one location to another - a process that is important in the release of neurotransmitter chemicals, the secretion of insulin and countless other duties.

James Rothman of Yale University in New Haven, Connecticut; Randy Schekman at the University of California, Berkeley; and Thomas Südhof of Stanford University in California, will split the prize - worth 8 million Swedish kronor (US $\$ 1.2$ million) - which will be awarded at a ceremony on 10 December in Stockholm.

"My first reaction was, 'Oh my God!'” Schekman says. "That was also my second reaction."
The Nobel committee recognized the three researchers "for their discoveries of machinery regulating vesicle traffic, a major transport system in our cells". Vesicles are tiny packages, enclosed by a layer of lipids, that ferry biomolecules around the cell by fusing with other structures.

Hidde Ploegh, an immunologist at the Whitehead Institute in Cambridge, Massachusetts, says that the trio's work is so fundamental to cell biology that it is easy to take it for granted. "When we teach cell biology to biology majors and graduate students, many aspects of vesicular transport are presented as if they've been there all along."

Rothman and Schekman began their work in the late 1970s, when scientists had recognized that vesicles were involved in cellular trafficking, but they knew little about how these structures helped to tame the inner chaos of the cell. To determine how cells make vesicles, how these packages know where to go and how they fuse with other membranes, the two took very different approaches.

Rothman, known for his methodical approach to biochemistry (and noted by some at conferences for his uncanny resemblance to King Henry VIII of England), began as a young researcher at Stanford with the goal of recreating the whole process in a test tube. The work involved looking for proteins by purifying extracts from mammalian cells, some of which had been infected with a virus that, like many other viruses, makes use of the vesicular transport system. One of the viral proteins was used as a marker to track progress through the vesicle system.

Rothman's team purified several proteins that were crucial for vesicles to fuse with their membrane targets. The first was named $\mathrm{N}$-ethylmaleimide-sensitive factor, or NSF.

Across San Francisco Bay in Berkeley, Schekman and his team were taking a more diverse approach to studying the same problem. The researchers mutated strains of the yeast Saccharomyces cerevisiae, and screened for the ones that could no longer shuttle certain enzymes around the cell. The work eventually turned up 23 'secretory genes', one of which turned out to encode the protein NSF.

Many scientists were initially sceptical of the findings, says Marino Zerial, a molecular biologist who studies cellular trafficking at the Max Planck Institute of Molecular Cell Biology and Genetics in Dresden, Germany. "The biochemists didn't like the genetic approach and geneticists were doubting the biochemical approach," he says.

But in subsequent work in the late 1980s and early 1990s, the laboratories of Schekman and Rothman worked out the basic mechanisms of vesicle fusion, in which proteins embedded in the membranes of vesicles recognize proteins on the membranes of their destination through a lock-and-key mechanism that is still being worked out. "The synergy that came out from these two is unbelievable," Zerial says. "They're my heroes."

Südhof's work, meanwhile, focused on vesicle fusion in neurons, which communicate with other neurons through neurotransmitter chemicals that are packaged in vesicles. Beginning in the late 1980s, Südhof and a team led by Richard Scheller - now at Genentech, a biotechnology company in 
- South San Francisco, California - identified a number of proteins that are important to the process. Südhof also showed how the fusion of neurotransmitter-containing vesicles is triggered by calcium.

"It's a fantastic story - it's how in a relatively short time frame, 25 or 30 years, we've gone from knowing almost nothing about how membrane trafficking occurs in cells to knowing the proteins and actors and having a reasonable idea of how they act," says William Wickner, a biochemist at Dartmouth Medical School in Hanover, New Hampshire. "These three are responsible for major, major advances."
Johannes Herrmann, a cell biologist at the Technical University of Kaiserslautern in Germany, was trained by Schekman and had nothing but praise for his former mentor. "It's a great day," he says. He remembers that Schekman would set grand goals for his laboratory and, when they were reached, reward the entire lab with banquet dinners. Schekman also encouraged his students to pursue outside interests, such as travel and the arts. It may not be surprising then, that Schekman served as the editor of The Proceedings of the National Academy of Sciences from 2006 until 2011, when he left to become editor-in-chief of the open-access journal eLife.

Ploegh says that the award also highlights the relevance of basic research to biomedicine. "This recognizes a fundamental discovery that I think was made without any urgent need to solve a medical problem."

All three laureates are previous winners of the Albert Lasker Basic Medical Research Award, which is often regarded as an indicator of future Nobel prizes in medicine. Südhof won it this year (along with Scheller) for his work on the release of neurotransmitters. Schekman and Rothman shared the award in 2002.

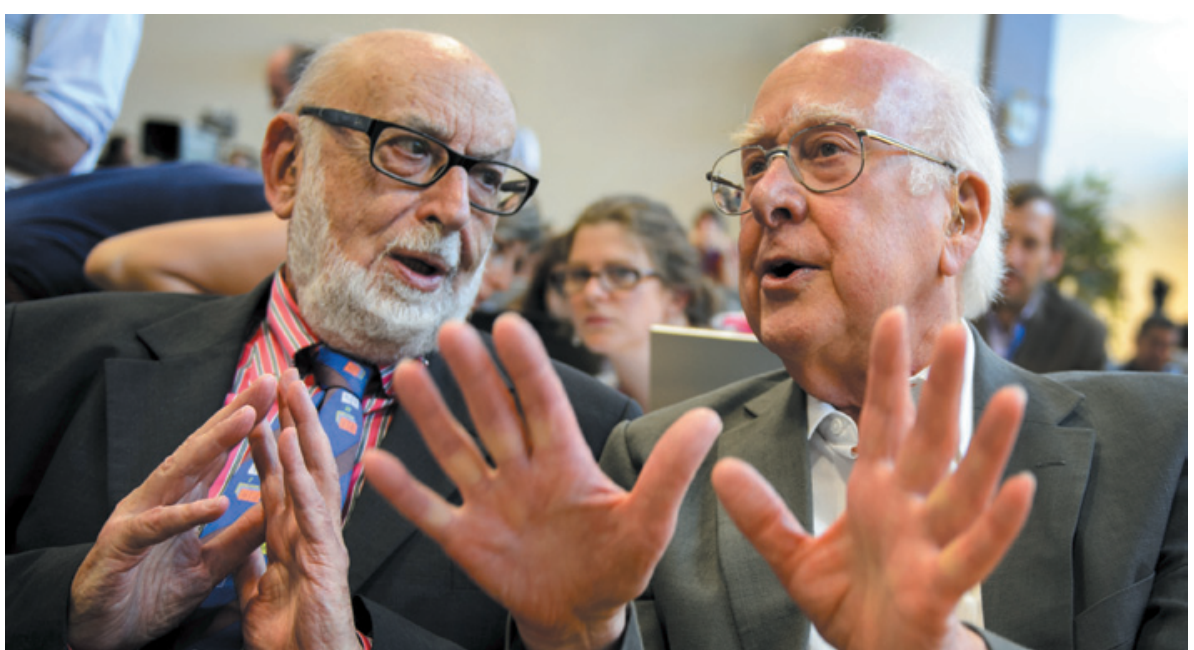

Nobel laureates Peter Higgs (right) and François Englert at CERN in July 2012.

\section{NOBEL PRIZE}

\section{Higgs theorists} amass physics prize

\section{François Englert and Peter Higgs rewarded with Nobel 50 years after hunt for boson began.}

\section{BY RICHARD VAN NOORDEN}

$\mathrm{T}$ housands of scientists were involved in hunting down the Higgs boson, this generation's greatest discovery in particle physics. But for the committee awarding the Nobel Prize in Physics, two names mattered most. In an announcement on 8 October in Stockholm, Peter Higgs of the University of Edinburgh, UK, and François Englert of the Free University of Brussels were named Nobel laureates for developing the theory of what is now commonly called the Higgs mechanism: the process by which a field pervading space gives other fundamental particles mass, and which implies the existence of the Higgs boson. Regarding the committee's choice, "I think in all honesty, this is what I would have done," says John Ellis, a theoretical physicist at CERN, Europe's particle-physics lab near Geneva, Switzerland.

The existence of the boson was announced to cheers at CERN on 4 July last year, after the particle was fleetingly produced in high-energy collisions at the lab's $€ 3$-billion (US\$4.1-billion) Large Hadron Collider. It would have been too complicated to try to honour the experimenters with the Nobel, says Ellis, who joined other CERN theorists in popping open champagne as the award was announced. "Englert and Higgs' pioneering work richly deserved this prize," he adds.

"I'm very, very happy to have the recognition of this extraordinary reward," says Englert. Higgs, who is notoriously modest and suffered a bout of bronchitis last month, made himself unavailable for interviews. The two winners had met for the first time at CERN last July.

The Higgs boson was the missing piece in the standard model of particle physics, which describes all known fundamental particles and forces, apart from gravity. The boson itself is the smallest possible ripple of the Higgs field, which gives mass to particles including electrons, quarks and the $\mathrm{W}$ and $\mathrm{Z}$ bosons that carry the weak nuclear force.

The idea was mooted in the 1960s, when physicists trying to describe the fundamental forces were wrestling with "embarrassing massless particles floating around in their theories", as Ellis puts it. In 1964, six physicists independently worked out how a field would resolve the problem. Robert Brout (who died in 2011) and Englert were the first to publish, in August 1964, followed three weeks later by Higgs - the only author, at the time, to allude to the heavy boson that the theory implied. Tom Kibble, Gerald Guralnik and Carl Hagen followed. "Almost nobody paid any attention," says Ellis - mostly because physicists were unsure how to make calculations using such theories. It was only after 1971, when Gerard 't Hooft sorted out the mathematics, that citations started shooting up and the quest for the Higgs began in earnest.

So numerous were the theorists involved, that Higgs reputedly referred to the ABEGHHK'tH (Anderson-Brout-Englert-Guralnik-HagenHiggs-Kibble-'t Hooft) mechanism. But that list of names is nothing compared with the legion of experimenters who joined the quest to track down the boson, with increasingly powerful particle accelerators that produced their own Nobel-prizewinning findings along the way.

"It's really an incredible thing that it's happened in my lifetime," Higgs told the audience at CERN when the particle was announced.

Alan Walker, a colleague of Higgs at Edinburgh, says, "That day was for the experimentalists. I guess today is for the theorists." - 